formation of concretions and continued absorption of the drug. Prolonged repeated use of activated charcoal after carbamazepine overdose in comatose patients is not beneficial and carries a hazard of aspiration.

\title{
VALPROATE-INDUCED CEREBRAL EDEMA
}

A case of cerebral edema resulting from acute sodium valproate poisoning in a 19 year old male is reported from Northwick Park and East Birmingham Hospitals, UK. On admission the patient was unconscious and the plasma valproate concentration was $900 \mathrm{mg} / \mathrm{L}$. He had taken $1200 \mathrm{mg}$ of sodium valproate along with $1500 \mathrm{mg}$ of aspirin. The salicylate blood level was $70 \mathrm{mg} / \mathrm{L}$. CT showed gross cerebral edema with slit-like ventricles and absence of cortical sulci and basal cisterns. Gastric lavage, fluid restriction and IV dexamethasone resulted in slow recovery. Acute complications included liver and renal dysfunction, hypocalcaemia and generalized muscle spasms. (Khoo SH, Leyland MJ. Cerebral edema following acute sodium valproate overdose. Clin Toxicol June 1992; 30:209-214.) (Reprints: Dr. S.H. Khoo, Monsall Hospital, Newton Health, Manchester, M108WR, UK.)

COMMENT. External leakage from feeding gastrostomies was reported in 4 of 8 children who received valproate sprinkle at the Hennepin County Medical Center, Minneapolis, MN (Jones-Saete C et al. Epilepsia July/Aug 1992; 33:692-695). Adherence of undissolved valproate particles to the exterior of the tube appeared to prevent the close approximation of the tube to the gastrostomies stoma. The problem was reduced by changing the tube more frequently or using a larger size tube.

\section{SEIZURE DISORDERS}

\section{PYRIDOXINE DEPENDENT EPILEPSY}

Four children with pyridoxine dependent seizures beginning at 2 to 19 months are reported from the Loyola University Medical Center, Maywood, IL. Patients were identified out of 51 treated routinely with pyridoxine for refractory seizures and seen over a 6 year period. The dose of pyridoxine was $50 \mathrm{mg}$ orally twice daily. The seizure types were atypical absence, myoclonic, generalized clonic and simple partial and complex partial. The authors suggest that pyridoxine should be tried in all children with seizure disorders with onset at any age who are poorly responsive to anticonvulsant drugs. (Coker SB, Postneonatal vitamin $B_{6}$ - dependent epilepsy. Pediatrics Aug 1992; 90:221-223.) (Reprints: S.B. Coker, M.D., Loyola University Medical Center, 2160 S. First Ave., Maywood, IL 60153.)

COMMENT. Pyridoxine dependent epilepsy may present after the neonatal period and may be manifested by many seizure types. Many of us have been discouraged by failure to uncover pyridoxine dependency in drug resistant epilepsies in children. Obviously, persistence rewards, but the relationship between pyridoxine and epilepsy is complex. 